\title{
Solitonic generation of five-dimensional black ring solution
}

\author{
Hideo Iguchi and Takashi Mishima \\ Laboratory of Physics, College of Science and Technology, Nihon University, \\ Narashinodai, Funabashi, Chiba 274-8501, Japan
}

(Dated: March 25, 2018)

\begin{abstract}
Using the solitonic solution-generating technique we rederived the one-rotational five-dimensional black ring solution found by Emparan and Reall. The seed solution is not the Minkowski metric, which is the seed of $S^{2}$-rotating black ring. The obtained solution has more parameters than the Emparan and Reall's $S^{1}$-rotating black ring. We found the conditions of parameters to reduce the solution to the $S^{1}$-rotating black ring. In addition we examined the relation between the expressions of the metric in the prolate-spheroidal coordinates and in the canonical coordinates.
\end{abstract}

PACS numbers: 04.50.+h, 04.20.Jb, 04.20.Dw, 04.70.Bw

One of the most important recent findings of the higher-dimensional General Relativity is a one-rotational black ring solution by Emparan and Reall 11. This solution is a vacuum, axially symmetric and asymptotically flat solution of the five-dimensional General Relativity. The topology of the event horizon is $S^{1} \times S^{2}$. The black ring rotates along the direction of the $S^{1}$. The extension of this solution to a two-rotational one has not yet been achieved.

Recently the present authors found a black ring solution with $S^{2}$ rotation by using a solitonic solutiongenerating technique 2]. In the analysis we reduced the problem to the four-dimensional one 3, 4, 5] and applied the formula [6] to obtain the metric functions. The seed solution of this ring is a simple Minkowski spacetime. Because the effect of rotation cannot compensate for the gravitational attractive force, the ring has a kind of strut structure. Figueras found a C-metric expression of $S^{2}$ rotating black ring solution [7]. Tomizawa et al. showed that the same black ring solution is obtained by using the inverse scattering method [8].

In this paper we generate the black ring with $S^{1}$ rotation by the solitonic solution-generating technique. We find that the seed solution is not a Minkowski spacetime. The obtained solution has more parameters than Emparan and Reall's black ring. It is therefore an extension of the result of Emparan and Reall and we need some additional conditions to reduce the solution we obtained to the black ring solution. In this analysis we use prolate-spheroidal coordinates. The relation between this and the canonical coordinates considered by Harmark 9] are analyzed. We also investigate the correspondence between the seed solutions and the solitonic ones from the viewpoints of rod structure. This viewpoint would be helpful to consider seed solutions for further new five-dimensional solutions. We cannot generate tworotational solutions by the solution-generating technique used here. However if we use another technique, e.g., inverse scattering method, for the seed solution used in this analysis or the seed with some corrections, the tworotational black ring solution may be obtained.

At first we briefly explain the procedure to generate axisymmetric solutions in the five-dimensional general rel- ativity. The spacetimes which we considered satisfy the following conditions: (c1) five dimensions, (c2) asymptotically flat spacetimes, (c3) the solutions of vacuum Einstein equations, (c4) having three commuting Killing vectors including time translational invariance and (c5) having a single non-zero angular momentum component. Under the conditions (c1) - (c5), we can employ the following Weyl-Papapetrou metric form (for example, see the treatment in 9]),

$$
\begin{aligned}
d s^{2}= & -e^{2 U_{0}}\left(d x^{0}-\omega d \phi\right)^{2}+e^{2 U_{1}} \rho^{2}(d \phi)^{2}+e^{2 U_{2}}(d \psi)^{2} \\
& +e^{2\left(\gamma+U_{1}\right)}\left(d \rho^{2}+d z^{2}\right),
\end{aligned}
$$

where $U_{0}, U_{1}, U_{2}, \omega$ and $\gamma$ are functions of $\rho$ and $z$. Then we introduce new functions $S:=2 U_{0}+U_{2}$ and $T:=U_{2}$ so that the metric form (1) is rewritten into

$$
\begin{aligned}
d s^{2}= & e^{-T}\left[-e^{S}\left(d x^{0}-\omega d \phi\right)^{2}+e^{T+2 U_{1}} \rho^{2}(d \phi)^{2}\right. \\
& \left.+e^{2\left(\gamma+U_{1}\right)+T}\left(d \rho^{2}+d z^{2}\right)\right]+e^{2 T}(d \psi)^{2}
\end{aligned}
$$

Using this metric form the Einstein equations are reduced to the following set of equations,

(i) $\nabla^{2} T=0$,

(ii) $\left\{\begin{aligned} \partial_{\rho} \gamma_{T} & =\frac{3}{4} \rho\left[\left(\partial_{\rho} T\right)^{2}-\left(\partial_{z} T\right)^{2}\right] \\ \partial_{z} \gamma_{T} & =\frac{3}{2} \rho\left[\partial_{\rho} T \partial_{z} T\right],\end{aligned}\right.$

(iii) $\nabla^{2} \mathcal{E}_{S}=\frac{2}{\mathcal{E}_{S}+\overline{\mathcal{E}}_{S}} \nabla \mathcal{E}_{S} \cdot \nabla \mathcal{E}_{S}$,

(iv) $\left\{\begin{aligned} \partial_{\rho} \gamma_{S} & =\frac{\rho}{2\left(\mathcal{E}_{S}+\overline{\mathcal{E}}_{S}\right)}\left(\partial_{\rho} \mathcal{E}_{S} \partial_{\rho} \overline{\mathcal{E}}_{S}-\partial_{z} \mathcal{E}_{S} \partial_{z} \overline{\mathcal{E}}_{S}\right) \\ \partial_{z} \gamma_{S} & =\frac{\rho}{2\left(\mathcal{E}_{S}+\overline{\mathcal{E}}_{S}\right)}\left(\partial_{\rho} \mathcal{E}_{S} \partial_{z} \overline{\mathcal{E}}_{S}+\partial_{\rho} \mathcal{E}_{S} \partial_{z} \overline{\mathcal{E}}_{S}\right),\end{aligned}\right.$

(v) $\left(\partial_{\rho} \Phi, \partial_{z} \Phi\right)=\rho^{-1} e^{2 S}\left(-\partial_{z} \omega, \partial_{\rho} \omega\right)$,

(vi) $\gamma=\gamma_{S}+\gamma_{T}$,

(vii) $U_{1}=-\frac{S+T}{2}$

where $\Phi$ is defined through the equation (v) and the function $\mathcal{E}_{\mathcal{S}}$ is defined by $\mathcal{E}_{S}:=e^{S}+i \Phi$. The most nontrivial task to obtain new metrics is to solve the equation (iii) because of its non-linearity. To overcome this 
difficulty here we use the method similar to the Neugebauer's Bäcklund transformation [10] or the HoenselaersKinnersley-Xanthopoulos transformation 11.

To write down the exact form of the metric functions, we follow the procedure given by Castejon-Amenedo and Manko [6]. In the five dimensional spacetime we start from the following form of a seed static metric

$$
\begin{aligned}
d s^{2}= & e^{-T^{(0)}}\left[-e^{S^{(0)}}\left(d x^{0}\right)^{2}+e^{-S^{(0)}} \rho^{2}(d \phi)^{2}\right. \\
& \left.+e^{2 \gamma^{(0)}-S^{(0)}}\left(d \rho^{2}+d z^{2}\right)\right]+e^{2 T^{(0)}}(d \psi)^{2} .
\end{aligned}
$$

For this static seed solution, $e^{S^{(0)}}$, of the Ernst equation (iii), a new Ernst potential can be written in the form

$$
\mathcal{E}_{S}=e^{S^{(0)}} \frac{x(1+a b)+i y(b-a)-(1-i a)(1-i b)}{x(1+a b)+i y(b-a)+(1-i a)(1-i b)},
$$

where $x$ and $y$ are the prolate spheroidal coordinates: $\rho=\sigma \sqrt{x^{2}-1} \sqrt{1-y^{2}}, z=\sigma x y$, with $\sigma>0$. The ranges of these coordinates are $1 \leq x$ and $-1 \leq y \leq 1$. The functions $a$ and $b$ satisfy the following simple firstorder differential equations

$$
\begin{aligned}
& (x-y) \partial_{x} a=a\left[(x y-1) \partial_{x} S^{(0)}+\left(1-y^{2}\right) \partial_{y} S^{(0)}\right], \\
& (x-y) \partial_{y} a=a\left[-\left(x^{2}-1\right) \partial_{x} S^{(0)}+(x y-1) \partial_{y} S^{(0)}\right], \\
& (x+y) \partial_{x} b=-b\left[(x y+1) \partial_{x} S^{(0)}+\left(1-y^{2}\right) \partial_{y} S^{(0)}\right] \\
& (x+y) \partial_{y} b=-b\left[-\left(x^{2}-1\right) \partial_{x} S^{(0)}+(x y+1) \partial_{y} S^{(0)}\right] .
\end{aligned}
$$

The metric functions for the five-dimensional metric (2) are obtained by using the formulas shown by [] $]$,

$$
\begin{aligned}
e^{S} & =e^{S^{(0)}} \frac{A}{B} \\
\omega & =2 \sigma e^{-S^{(0)}} \frac{C}{A}+C_{1} \\
e^{2 \gamma} & =C_{2}\left(x^{2}-1\right)^{-1} A e^{2 \gamma^{\prime}},
\end{aligned}
$$

where $C_{1}$ and $C_{2}$ are constants and $A, B$ and $C$ are given by

$$
\begin{aligned}
A:= & \left(x^{2}-1\right)(1+a b)^{2}-\left(1-y^{2}\right)(b-a)^{2}, \\
B:= & {[(x+1)+(x-1) a b]^{2}+[(1+y) a+(1-y) b]^{2}, } \\
C:= & \left(x^{2}-1\right)(1+a b)[(1-y) b-(1+y) a] \\
& \quad+\left(1-y^{2}\right)(b-a)[x+1-(x-1) a b] .
\end{aligned}
$$

In addition the $\gamma^{\prime}$ in Eq. (6) is a $\gamma$ function corresponding to the static metric,

$$
\begin{aligned}
d s^{2}= & e^{-T^{(0)}}\left[-e^{2 U_{0}^{(\mathrm{BH})}+S^{(0)}}\left(d x^{0}\right)^{2}+e^{-2 U_{0}^{(\mathrm{BH})}-S^{(0)}} \rho^{2}(d \phi)^{2}\right. \\
& \left.+e^{2\left(\gamma^{\prime}-U_{0}^{(\mathrm{BH})}\right)-S^{(0)}}\left(d \rho^{2}+d z^{2}\right)\right]+e^{2 T^{(0)}}(d \psi)^{2}
\end{aligned}
$$

where $U_{0}^{(\mathrm{BH})}=\frac{1}{2} \ln \left(\frac{x-1}{x+1}\right)$. And then the function $T$ is equals to $T^{(0)}$ and $U_{1}$ is given by the Einstein equation (vii).

Using the solution-generating technique described above, we construct the $S^{1}$-rotating black ring solution obtained by Emparan and Reall. The most important point is to find the seed metric of the black ring solution. To do so, it is useful to use the rod structures which was studied for the higher-dimensional Weyl solutions by Emparan and Reall 12 and for the nonstatic solutions by Harmark [9]. Using this rod structure analysis, we found the seed metric of the $S^{1}$-rotating black ring solution by analogy with the relations between the $S^{2}$-rotating black ring and its seed metric. (See [9] for the definition of the rod structure.)

We show the schematic pictures of rod structures of the $S^{2}$-rotating black ring and its seed solution in Fig. 10 2]. Through the solitonic transformation the segment $[-\sigma, \sigma]$ of semi-infinite spacelike rod which corresponds to the $\phi$ axis is changed to the finite timelike rod. To indicate that the $x^{0}$ and $\phi$ components of the eigenvector are not zero, we put the finite rod between $x^{0}$ and $\phi$ axes in Fig. [1] In the resulting solution this segment corresponds to the event horizon with $\phi$-rotation.

The rod structure of $S^{1}$-rotating black ring was investigated by Harmark [9]. There are two semi-infinite spacelike rods in the directions of $\partial / \partial \psi$ and $\partial / \partial \phi$. Note that these two semi-infinite spacelike rods assure the asymptotic flatness of the spacetime. Also there is a finite spacelike rod in $\partial / \partial \psi$ direction. A finite timelike rod has finite and semi-infinite spacelike rods in $\partial / \partial \psi$ direction on each side. This timelike rod corresponds to an event horizon with $\phi$-rotation.

Now we construct the seed solution for the $S^{1}$-rotating black ring. For this purpose we trace back from the rod structure of $S^{1}$-rotating black ring to the seed solution referring the analysis of $S^{2}$-rotating black ring. This can be achieved when we change the finite timelike rod to the finite spacelike rod in the $\partial / \partial \phi$ direction. In Fig. 2] we show the schematic pictures of these two rod structures.

The seed metric of $S^{1}$-rotating black ring is summarized as follows. The $\psi-\psi$ componet of the metric of black ring and its seed are exactly same as each other. Also the $0-0$ component of the seed metric is -1 . The seed functions of $S^{1}$-rotating black ring solution are obtained as

$$
S^{(0)}=T^{(0)}=\tilde{U}_{\lambda \sigma}+\tilde{U}_{-\eta_{1} \sigma}-\tilde{U}_{\eta_{2} \sigma},
$$

where the function $\tilde{U}_{d}$ is defined as $\tilde{U}_{d}$ := $\frac{1}{2} \ln \left[R_{d}+(z-d)\right]$ and $R_{d}=\sqrt{\rho^{2}+(z-d)^{2}}$. We assume that the parameters $\lambda, \eta_{1}$ and $\eta_{2}$ should satisfy the following inequalities

$$
1 \leq \lambda, \quad-1 \leq \eta_{1} \leq 1, \quad 1 \leq \eta_{2} \leq 1, \quad 0 \leq \eta_{1}+\eta_{2},
$$

to generate the black ring solution because the timelike rod appears in the region $-\sigma \leq z \leq \sigma$ after the solitonic 


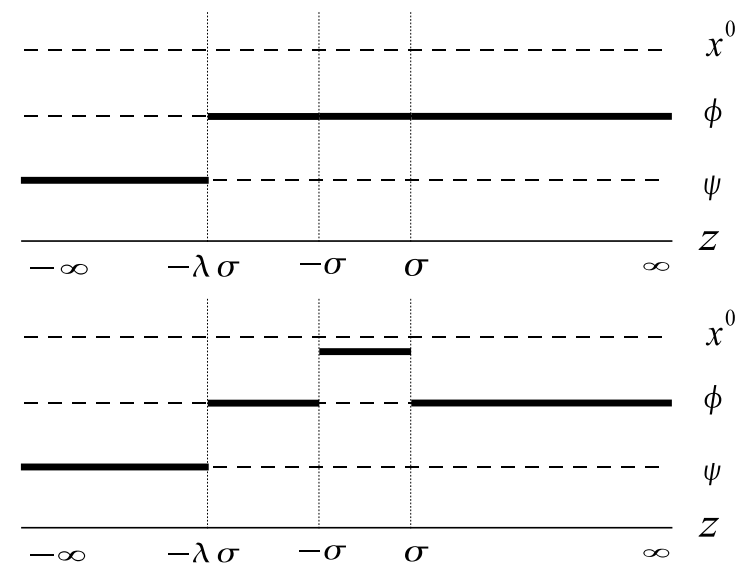

FIG. 1: Schematic pictures of rod structures. The upper panel shows the rod structure of Minkowski spacetime, which is a seed of $S^{2}$-rotating black ring. The lower panel shows the rod structure of the $S^{2}$-rotating black ring. The segment $[-\sigma, \sigma]$ of semi-infinite rod in the upper panel is tranformed to the finite timelike rod with $\phi$-rotation by the solution-generating transformation. The eigenvector of the finite timelike rod in the lower panel has non-zero $\phi$ component. Therefore we put this rod between $x^{0}$ and $\phi$ axes.

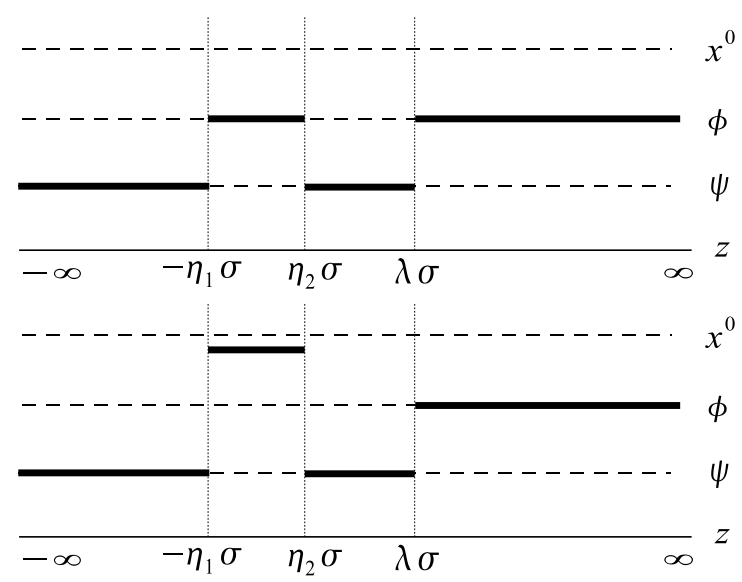

FIG. 2: Schematic pictures of rod structures. The upper panel shows the rod structure of seed metric of $S^{1}$-rotating black ring. The lower panel shows the rod structure of $S^{1}$ rotating black ring. The finite spacelike $\operatorname{rod}\left[-\eta_{1} \sigma, \eta_{2} \sigma\right]$ in the upper panel is altered to the finite timelike rod by the solution-generating transformation.

transformation. Under these assumptions, the region of $x=1$ and $-\eta_{1}<y<\eta_{2}$ of the solitonic solution corresponds to the event horizon. Also the region of $x>\lambda$ and $y=1$ is the fixed points of the $\phi$ rotation. The regions of $1<x<\lambda$ and $y=1, x=1$ and $\eta_{2}<y<1, x=1$ and $-1<y<-\eta_{1}$, and $y=-1$ become fixed points of $\psi$ rotation in the black ring spacetime.

Substituting the seed function (8) into the differential equations (3), we obtain the solutions of these equations as,

$$
\begin{aligned}
& a=\frac{\alpha}{2 \sigma^{\frac{1}{2}}} \frac{e^{2 U_{\sigma}}+e^{2 \tilde{U}_{\lambda \sigma}}}{e^{\tilde{U}_{\lambda \sigma}}} \frac{e^{2 U_{\sigma}}+e^{2 \tilde{U}_{-\eta_{1} \sigma}}}{e^{\tilde{U}_{-\eta_{1} \sigma}}} \frac{e^{\tilde{U}_{\eta_{2} \sigma}}}{e^{2 U_{\sigma}}+e^{2 \tilde{U}_{\eta_{2} \sigma}}}, \\
& b=2 \sigma^{\frac{1}{2}} \beta \frac{e^{\tilde{U}_{\lambda \sigma}}}{e^{2 U_{-\sigma}+e^{2 \tilde{U}_{\lambda \sigma}}} \frac{e^{\tilde{U}_{-\eta_{1} \sigma}}}{e^{2 U_{-\sigma}}+e^{2 \tilde{U}_{-\eta_{1} \sigma}}} \frac{e^{2 U_{-\sigma}}+e^{2 \tilde{U}_{\eta_{2} \sigma}}}{e^{\tilde{U}_{\eta_{2} \sigma}}},}
\end{aligned}
$$

where $\alpha$ and $\beta$ are integration constants and $U_{c}:=$ $\frac{1}{2} \ln \left[R_{c}-(z-c)\right]$.

Next we reduce the explicit expression for the $\gamma^{\prime}$. Read out the functions $S^{\prime}$ and $T^{\prime}$ from Eq. (7) as

$$
\begin{aligned}
S^{\prime} & =2 U_{0}^{(B H)}+S^{(0)} \\
& =2\left(\tilde{U}_{\sigma}-\tilde{U}_{-\sigma}\right)+\tilde{U}_{\lambda \sigma}+\tilde{U}_{-\eta_{1} \sigma}-\tilde{U}_{\eta_{2} \sigma} \\
T^{\prime} & =T^{(0)}=\tilde{U}_{\lambda \sigma}+\tilde{U}_{-\eta_{1} \sigma}-\tilde{U}_{\eta_{2} \sigma},
\end{aligned}
$$

and substitute them into

$\partial_{\rho} \gamma^{\prime}=\frac{1}{4} \rho\left[\left(\partial_{\rho} S^{\prime}\right)^{2}-\left(\partial_{z} S^{\prime}\right)^{2}\right]+\frac{3}{4} \rho\left[\left(\partial_{\rho} T^{\prime}\right)^{2}-\left(\partial_{z} T^{\prime}\right)^{2}\right]$,

$$
\partial_{z} \gamma^{\prime}=\frac{1}{2} \rho\left[\partial_{\rho} S^{\prime} \partial_{z} S^{\prime}\right]+\frac{3}{2} \rho\left[\partial_{\rho} T^{\prime} \partial_{z} T^{\prime}\right],
$$

so we can confirm that $\gamma^{\prime}$ is divided as

$$
\begin{aligned}
\gamma^{\prime}= & \gamma_{\sigma, \sigma}^{\prime}+\gamma_{-\sigma,-\sigma}^{\prime}+\gamma_{\lambda \sigma, \lambda \sigma}^{\prime}+\gamma_{-\eta_{1} \sigma,-\eta_{1} \sigma}^{\prime}+\gamma_{\eta_{2} \sigma, \eta_{2} \sigma}^{\prime} \\
& -2 \gamma_{\sigma,-\sigma}^{\prime}+\gamma_{\sigma, \lambda \sigma}^{\prime}+\gamma_{\sigma,-\eta_{1} \sigma}^{\prime}-\gamma_{\sigma, \eta_{2} \sigma}^{\prime} \\
& -\gamma_{-\sigma, \lambda \sigma}^{\prime}-\gamma_{-\sigma,-\eta_{1} \sigma}^{\prime}+\gamma_{-\sigma, \eta_{2} \sigma}^{\prime}+2 \gamma_{\lambda \sigma,-\eta_{1} \sigma}^{\prime} \\
& -2 \gamma_{\lambda \sigma, \eta_{2} \sigma}^{\prime}-2 \gamma_{-\eta_{1} \sigma, \eta_{2} \sigma}^{\prime},
\end{aligned}
$$

where $\gamma_{c d}^{\prime}$ satisfies the following equations

$$
\begin{aligned}
& \partial_{\rho} \gamma_{c d}^{\prime}=\rho\left[\partial_{\rho} \tilde{U}_{c} \partial_{\rho} \tilde{U}_{d}-\partial_{z} \tilde{U}_{c} \partial_{z} \tilde{U}_{d}\right], \\
& \partial_{z} \gamma_{c d}^{\prime}=\rho\left[\partial_{\rho} \tilde{U}_{c} \partial_{z} \tilde{U}_{d}+\partial_{\rho} \tilde{U}_{d} \partial_{z} \tilde{U}_{c}\right] .
\end{aligned}
$$

These equations (9) and (10) have the solution,

$$
\gamma_{c d}^{\prime}=\frac{1}{2} \tilde{U}_{c}+\frac{1}{2} \tilde{U}_{d}-\frac{1}{4} \ln Y_{c d},
$$

where $Y_{c d}:=R_{c} R_{d}+(z-c)(z-d)+\rho^{2}$.

Now the functions which is needed to express the full metric are completely obtained. The full metric is expressed as

$$
\begin{aligned}
d s^{2}= & -\frac{A}{B}\left[d x^{0}-\left(2 \sigma e^{-S^{(0)}} \frac{C}{A}+C_{1}\right) d \phi\right]^{2} \\
& +\frac{B}{A} e^{-2 S^{(0)}} \rho^{2}(d \phi)^{2}+e^{2 S^{(0)}}(d \psi)^{2} \\
& +C_{2} \sigma^{2} \frac{x^{2}-y^{2}}{x^{2}-1} B e^{2\left(\gamma^{\prime}-S^{(0)}\right)}\left(\frac{d x^{2}}{x^{2}-1}+\frac{d y^{2}}{1-y^{2}}\right) .
\end{aligned}
$$


In the following the constants $C_{1}$ and $C_{2}$ are fixed as

$$
C_{1}=\frac{2 \sigma^{1 / 2} \alpha}{1+\alpha \beta}, \quad C_{2}=\frac{1}{\sqrt{2}(1+\alpha \beta)^{2}},
$$

to assure that the spacetime asymptotes to a fivedimensional Minkowski spacetime globally. We can confirm this by taking the asymptotic limit, $x \rightarrow \infty$, of the metric.

The above solution is an extension of $S^{1}$-rotating black ring solution because it contains singular cases. In general the spacetime described by the metric (11) includes some harmful regions, for example, the region where closed timelike curves exist. In fact, the metric component $g_{\phi \phi}$ becomes negative around $(x, y)=(1,1)$ and $(1,-1)$. These singular behaviors are cured by setting the parameters $\alpha$ and $\beta$ as

$$
\alpha=\sqrt{\frac{2\left(1-\eta_{2}\right)}{(\lambda-1)\left(1+\eta_{1}\right)}}, \beta=\sqrt{\frac{(\lambda+1)\left(1-\eta_{1}\right)}{2\left(1+\eta_{2}\right)}} .
$$

The asymptotic form of $\mathcal{E}_{S}$ near the infinity $\tilde{r}=\infty$ becomes

$$
\begin{aligned}
\mathcal{E}_{S}= & \tilde{r} \cos \theta\left[1-\frac{\sigma}{\tilde{r}^{2}} \frac{P(\alpha, \beta, \lambda)}{(1+\alpha \beta)^{2}}+\cdots\right] \\
& +2 i \sigma^{1 / 2}\left[\frac{\alpha}{1+\alpha \beta}-\frac{2 \sigma \cos ^{2} \theta}{\tilde{r}^{2}} \frac{Q(\alpha, \beta, \lambda)}{(1+\alpha \beta)^{3}}+\cdots\right],
\end{aligned}
$$

where we introduced the new coordinates $\tilde{r}$ and $\theta$ through the relations

$$
x=\frac{\tilde{r}^{2}}{2 \sigma}+\lambda-\eta_{1}-\eta_{2}, y=\cos 2 \theta,
$$

and

$$
\begin{aligned}
P(\alpha, \beta, \lambda)= & 4\left(1+\alpha^{2}-\alpha^{2} \beta^{2}\right) \\
Q(\alpha, \beta, \lambda)= & \alpha\left(2 \alpha^{2}-\eta_{1}-\eta_{2}+\lambda+3\right)-2 \alpha^{2} \beta^{3} \\
& -\beta\left[2(2 \alpha \beta+1)\left(\alpha^{2}+1\right)\right. \\
& \left.+\left(\eta_{1}+\eta_{2}-\lambda-1\right) \alpha^{2}(\alpha \beta+2)\right] .
\end{aligned}
$$

From the asymptotic behavior of the Ernst potential, we can compute the mass parameter $m^{2}$ and rotational parameter $m^{2} a_{0}$ as

$$
m^{2}=\sigma \frac{P(\alpha, \beta, \lambda)}{(1+\alpha \beta)^{2}}, \quad m^{2} a_{0}=4 \sigma^{3 / 2} \frac{Q(\alpha, \beta, \lambda)}{(1+\alpha \beta)^{3}} .
$$

For the black ring solution we obtain

$$
\begin{aligned}
m^{2} & =\frac{8 \sigma\left(\eta_{1}+\eta_{2}\right)\left(\lambda-\eta_{2}\right)}{(\lambda-1)\left(1+\eta_{1}\right)\left(1+\eta_{2}\right)(1+\alpha \beta)^{2}} \\
m^{2} a_{0} & =m^{2} \frac{\sqrt{\sigma}\left(\alpha\left(1+\lambda \eta_{1}\right)-2 \beta \eta_{2}\right)}{1+\alpha \beta}
\end{aligned}
$$

where we use the Eq. (12). From Eqs. (13) and (14) we can obtain the usefull relation

$$
\frac{a_{0}^{2}}{m^{2}}=\frac{\left(\left(1+\lambda \eta_{1}\right) \sqrt{1-\eta_{2}^{2}}-\eta_{2} \sqrt{\left(\lambda^{2}-1\right)\left(1-\eta_{1}^{2}\right)}\right)^{2}}{4\left(\lambda-\eta_{2}\right)\left(\eta_{1}+\eta_{2}\right)}
$$

When $\eta_{1}=\eta_{2}=1$ the black ring becomes static because $\alpha=\beta=0$ from Eq. (12). The one-rotational black hole limit [13] of the black ring is realized when we set

$$
\lambda=1+\epsilon, \quad \eta_{2}=1-k \epsilon,
$$

where $k>0$ is constant, and then, take the limit $\epsilon \rightarrow 0$.

The periods of $\phi$ and $\psi$ are defined as

$$
\Delta \phi=2 \pi \lim _{\rho \rightarrow 0} \sqrt{\frac{\rho^{2} g_{\rho \rho}}{g_{\phi \phi}}} \text { and } \Delta \psi=2 \pi \lim _{\rho \rightarrow 0} \sqrt{\frac{\rho^{2} g_{\rho \rho}}{g_{\psi \psi}}}
$$

to avoid a conical singularity. We see that the period of $\phi$ is $\Delta \phi=2 \pi$ and the period of $\psi$ is $\Delta \psi=2 \pi$ outside the ring and

$$
\Delta \psi=2 \pi \frac{\left(\frac{\lambda-\eta_{2}}{\lambda+\eta_{1}}\right)\left(1+\sqrt{\frac{(\lambda-1)\left(1-\eta_{1}\right)\left(1-\eta_{2}\right)}{(\lambda+1)\left(1+\eta_{1}\right)\left(1+\eta_{2}\right)}}\right)}{\sqrt{\frac{\lambda-1}{\lambda+1}}+\sqrt{\frac{\left(1-\eta_{1}\right)\left(1-\eta_{2}\right)}{\left(1+\eta_{1}\right)\left(1+\eta_{2}\right)}}},
$$

inside the ring. In general there is a conical singularity inside or outside the ring. This conical singularity is cured by setting the parameters $\lambda, \eta_{1}$ and $\eta_{2}$ to satisfy the relation

$$
\begin{aligned}
& \left(\frac{\lambda-\eta_{2}}{\lambda+\eta_{1}}\right)\left(1+\sqrt{\frac{(\lambda-1)\left(1-\eta_{1}\right)\left(1-\eta_{2}\right)}{(\lambda+1)\left(1+\eta_{1}\right)\left(1+\eta_{2}\right)}}\right) \\
& =\sqrt{\frac{\lambda-1}{\lambda+1}}+\sqrt{\frac{\left(1-\eta_{1}\right)\left(1-\eta_{2}\right)}{\left(1+\eta_{1}\right)\left(1+\eta_{2}\right)}} .
\end{aligned}
$$

Finaly we consider the coordinates transformation between the prolate-spheroidal coordinates used here and the canonical coordinates analyzed by Harmark [9]. See 9] for the notaiton and the exact expression of the metric in the canonical coordinates. We however use $\tilde{\rho}$ and $\tilde{z}$ for $\rho$ and $z$ of $[9]$.

Comparing the functional forms of $\psi-\psi$ components, we obtain the relations between these two coordinates. These two coordinates can be transformed into each other through the relation

$$
\begin{aligned}
& \tilde{\rho}=\rho, \\
& \tilde{z}=z+\frac{\eta_{1}-\eta_{2}}{2} \sigma=\sigma\left(x y+\frac{\eta_{1}-\eta_{2}}{2}\right) .
\end{aligned}
$$

In addition, the parameters should satisfy the following relations,

$$
\begin{aligned}
\kappa^{2} & =\sigma\left(\lambda+\frac{\eta_{1}-\eta_{2}}{2}\right) \\
c & =\frac{\eta_{1}+\eta_{2}}{2 \lambda+\eta_{1}-\eta_{2}} \\
b & =\frac{(\lambda+1+(\lambda-1) \alpha \beta)^{2}-\left(\lambda^{2}-1\right)(1+\alpha \beta)^{2}}{(\lambda+1+(\lambda-1) \alpha \beta)^{2}+\left(\lambda^{2}-1\right)(1+\alpha \beta)^{2}}
\end{aligned}
$$

to assure the equivalence of these two expressions. Here the parameters $\alpha$ and $\beta$ satisfy the conditions (12). Also we have to rescale the $\rho-z$ part of the metric as

$$
e^{2 \nu}=\frac{1-b}{(1-c)^{2}} e^{2\left(\gamma+U_{1}\right)}
$$


Note that $b \geq c$ when $\lambda \geq 1, \eta_{1} \leq 1$ and $\eta_{2} \leq 1$. From the static black ring condition $b=c[9]$, we can derive the following relation

$$
\frac{1-\eta_{2}}{1+\eta_{2}}=\left(\frac{1-\eta_{1}}{1+\eta_{1}}\right)\left(\frac{\lambda-1}{\lambda+1}\right)
$$

which holds when $\eta_{1}=\eta_{2}=1$. Indeed the black ring is static in this case because $\alpha=\beta=0$ from Eq. (12). The black ring solution becomes one-rotational black hole when we take the limits $b, c \rightarrow 1[9]$. These limits surely correspond to the limits $\lambda, \eta_{2} \rightarrow 1$.

There are six parameters $\left(\lambda, \eta_{1}, \eta_{2}, \sigma, \alpha, \beta\right)$ in the metric (11) with two relations (12). While there are three parameters $(b, c, \kappa)$ in the canonical coordinates. The parameter $\sigma$ appears only in the relation of $\kappa^{2}$, Eq. (17), and contributes to the scaling of the coordinates. Thus we can freely fix one of parameters $\left(\lambda, \eta_{1}, \eta_{2}\right)$. Here we set $\eta_{1}=1$ because the relations obtained above become simple. In this case we can inversely solve Eqs. (17) (19). The results are

$$
\eta_{1}=1, \eta_{2}=\frac{2 c+c b-b}{(1+c) b}, \lambda=\frac{1}{b}, \sigma=\frac{1+c}{1+b} b \kappa^{2} .
$$

When the black ring does not have a conical singularity, these relations become

$$
\eta_{1}=1, \eta_{2}=c, \lambda=\frac{1+c^{2}}{2 c}, \sigma=\frac{2 c}{1+c} \kappa^{2},
$$

and the parameter $\eta_{2}$ lies in the range $0<\eta_{2} \leq 1$.

Next we consider the relations between physical variables. The ADM mass and angular momentum of the black ring were derived by Emparan and Reall [1] as

$$
M=\frac{3 \pi \kappa^{2} b}{2(1-c)}, \quad J_{1}=\sqrt{2} \pi \kappa^{3} \frac{\sqrt{b(b-c)(1+b)}}{(1-c)^{2}} .
$$

These two variables are related to the mass and rotational parameters as

$$
m^{2}=\frac{8(1-c)^{2}}{3 \pi(1-b)} M, \quad m^{2} a_{0}=\frac{4}{\pi} \frac{(1-c)^{3}}{(1-b)^{\frac{3}{2}}} J_{1} .
$$

Using the balanced black ring conditions (16) with $\eta_{1}=$ 1, the right hand side of Eq. (15) can be reduced to the following form,

$$
\frac{a_{0}^{2}}{m^{2}}=\frac{\left(1+\eta_{2}\right)^{3}}{8 \eta_{2}}=\frac{27 \pi}{32} \frac{J_{1}^{2}}{M^{3}} .
$$

This agrees with the previous results [1].

In this paper we rederived the $S^{1}$-rotating black ring solution by the solitonic slution-generating technique. Using the rod structure analysis we found the seed solution of the black ring on the analogy of the relation between the $S^{2}$-rotating black ring and its seed solution. The relations between the seed and obtained solitonic solutions can be easily understood through the analysis of their rod structures. Thus the rod structure analysis is expected to be a useful guide to construct seed solutions for new solutions. In addition we obtained the relations between the prolate-spheroidal coordinates and the canonical coordinates. This means that using the coordinates transformation between canonical and Cmetric coordinates obtained by Harmark 9 ], the prolatespheroidal coordinates also can be transformed into the C-metric coordinates.

As is the $S^{2}$-rotating black ring [8], the $S^{1}$-rotating black ring solution would be generated from the seed solution obtained here by the inverse scattering method. Also it might be expected that the two-rotational black ring is obtained from the seed solution or the seed with some deformations by this method.

This work is partially supported by Grant-in-Aid for Young Scientists (B) (No. 17740152) from Japanese Ministry of Education, Science, Sports, and Culture and by Nihon University Individual Research Grant for 2005.
[1] R. Emparan and H. S. Reall, Phys. Rev. Lett. 88, 101101 (2002).

[2] T. Mishima and H. Iguchi, Phys. Rev. D 73, 044030 (2006); H. Iguchi and T. Mishima, hep-th/0605090

[3] P. O. Mazur and L. Bombelli, J. Math. Phys. 28, 406 (1987).

[4] T. Dereli, A. Eriş and A. Karasu, Nuovo Cimento B 93, 102 (1986).

[5] W. Bruckman, Phys. Rev. D 34, 2990 (1986).

[6] J. Castejon-Amenedo and V. S. Manko, Phys. Rev. D 41, 2018 (1990).

[7] P. Figueras, J. High Energy Phys. 07 (2005) 039.
[8] S. Tomizawa, Y. Morisawa and Y. Yasui, Phys. Rev. D 73, 064009 (2006).

[9] T. Harmark, Phys. Rev. D 70, 124002 (2004).

[10] G. Neugebauer, J. Phys. A 13, L19 (1980).

[11] C. Hoenselaers, W. Kinnersley and B. C. Xanthopoulos, J. Math. Phys. 20, 2530 (1979).

[12] R. Emparan and H. S. Reall, Phys. Rev. D 65, 084025 (2002).

[13] R. C. Myers and M. J. Perry, Annals Phys. 172, 304 (1986). 